\title{
Quedas em idosos e comorbidades clínicas
}

\author{
Falls in the elderly and clinical comorbidities
}

\section{Caídas en adultos mayores y comorbilidades clínicas}

\author{
Daniele A. Lima, Vania O. B. Cezario
}

\begin{abstract}
Resumo
Queda pode ser definida como um deslocamento não intencional do corpo para um nível inferior à posição inicial, com incapacidade de correção em tempo hábil. As quedas entre pessoas idosas constituem um grave problema de saúde pública devido às complicações que acarretam à saúde do idoso e ao alto custo decorrente em assistência, tanto em nível hospitalar quanto domiciliar. A perda da autonomia e da independência do idoso, a presença de fraturas, a restrição de atividades e a imobilidade associadas a quedas acarretam um aumento do risco de institucionalização. Esse desfecho aumenta o declínio funcional, leva a problemas psicológicos, como a depressão, o medo de sofrer novas quedas e o risco de morte, além de prejuízos sociais relacionados à família. Muitos são os fatores responsáveis pelo desenvolvimento desses eventos, sendo necessário conhecer os seus fatores de risco, tanto intrínsecos quanto extrínsecos, para que se possa planejar e implementar medidas de prevenção. O objetivo desse artigo foi analisar os fatores de risco associados a quedas em idosos. Utilizou-se o método de revisão da literatura, tendo como fontes de pesquisa periódicos, teses e artigos científicos indexados em bases eletrônicas de dados. Os fatores encontrados na literatura mais comumente associados às quedas em idosos foram: idade avançada, sedentarismo, maior número de medicações de uso contínuo, uso de benzodiazepínicos, presença de doenças crônicas - inclusive as demenciais -, comprometimento da acuidade visual, antecedentes de quedas, autopercepção de saúde ruim, depressão, obstáculos no domicílio, presença de alterações de marcha e de equilíbrio. Concluímos que o conhecimento dos fatores de risco é importante para nortear o planejamento de medidas preventivas com o intuito de evitar quedas e suas consequências, e tendo como objetivo principal a redução do declínio funcional e a melhora da qualidade de vida da população idosa.
\end{abstract}

Descritores: Idosos; Fatores de risco; Quedas; Controle postural; Marcha.

\begin{abstract}
Falls can be defined as unintentional displacements of the body to a level below the starting position due to the inability to be corrected in timely manner. Falls among the elderly are a major public health problem due to complications that can cause health problems and the high costs for health assistance both for the hospital as well as the family. Loss of autonomy, of independence, the presence of fractures, restricted activity and immobility may cause increased risk of institutionalization of the elderly. This outcome increases functional decline, may lead to psychological problems such as depression, fear of suffering new falls and the risk of death as well as social impairments related to the family. Many factors are responsible for the development of such events and it is necessary to know both intrinsic and extrinsic risk factors to plan and implement measures to prevent falls among the elderly. This paper aims to identify the risk factors associated with falls among the elderly. We used the literature review method and as research sources: journals, theses and scientific articles indexed in electronic databases. The most commonly found factors in the literature associated with falls among the elderly were: advanced age, sedentary lifestyle, a greater number of the use of continuous medications, the use of benzodiazepine, the presence of chronic diseases - including dementia -, impaired visual acuity, history of falls, self-perception of poor health, depression, obstacles in the household, presence of abnormal gait and balance. We conclude that knowledge of risk factors is important to guide the planning of preventive measures and public policies to avoid falls and their consequences, aiming the reduction of functional decline and improvement of the quality of life of the elderly population.
\end{abstract}

Keywords: Aged; Risk factors; Falls; Postural control; Gait. 


\section{Resumen}

La caída puede ser definida como un desplazamiento no intencional del cuerpo a un nivel inferior de la posición inicial, con incapacidad de corrección en tiempo hábil. Las caídas entre personas adultas mayores constituyen un grave problema de salud pública debido a las complicaciones que causan en la salud del enfermo y al alto costo resultante de la asistencia, tanto a nivel hospitalario como domiciliar. La pérdida de la autonomía y de la independencia del adulto mayor, la presencia de fracturas, la restricción de actividades y la inmovilidad asociadas a caídas, conducen a un aumento del riesgo de institucionalización. Este desenlace aumenta el declive funcional, lleva a problemas psicológicos, como la depresión, el miedo a sufrir nuevas caídas y el riesgo de muerte, además de perjuicios sociales relacionados con la familia. Muchos factores son responsables del desarrollo de estos sucesos, siendo necesario conocer los factores de riesgo, tanto intrínsecos como extrínsecos, para que se pueda planear e implementar medidas de prevención. El objetivo de este artículo fue analizar los factores de riesgo asociados a caídas en adultos mayores. Se utilizó el metodo de revisión de la literatura, teniendo como fuentes de investigación: periódicos, tesis y artículos científicos indexados en bases electrónicas de datos. Los factores encontrados en la literatura comúnmente asociados a las caídas en adultos mayores fueron: edad avanzada, sedentarismo, mayor número de medicaciones de uso continuo, uso de benzodiazepínicos, presencia de enfermedades crónicas, incluso las demenciales, comprometimiento de la acuidad visual, antecedente de caídas, autopercepción de mala salud, depresión, obstáculos en el domicilio, presencia de alteraciones de la marcha y del equilibrio. Concluimos que el conocimiento de los factores de riesgo es importante para orientar la planificación de medidas preventivas con el objetivo de evitar caídas y sus consecuencias, teniendo como objetivo principal la reducción del declive funcional y la mejora de la calidad de vida de la población adulta mayor.

Palabras clave: Personas mayores; Factores de riesgo; Caídas; Postura; Marcha.

\section{Introdução}

As quedas ocorrem em virtude da perda súbita do equilíbrio postural. A queda pode ser considerada uma síndrome geriátrica, dado que geralmente é um evento multifatorial e heterogêneo. Além disso, a queda pode ser um fator preditor de que algo está errado com a saúde do idoso, podendo indicar a eminência de uma doença ainda não diagnosticada e associada a um pior prognóstico.' O custo social da queda é imenso e se torna maior quando o idoso tem diminuição da autonomia e da independência. $O$ número crescente de quedas com o aumento da idade está bem documentado pela literatura científica: entre 65 e 74 anos, a taxa de quedas é de 32\%; entre 75 e 84 anos, 35\%; e acima de 85 anos, $51 \%{ }^{2}$

O envelhecimento acarreta alterações fisiológicas que favorecem a ocorrência de quedas, como declínio da força muscular, alterações na massa óssea, deficit de equilíbrio, lentificação do tempo de reação, aumento do balanço do corpo, declínio dos reflexos, redução do controle postural, da coordenação motora, da flexibilidade, além de deficiências de visão, propriocepção e do sistema vestibular. ${ }^{3,4}$ Há um declínio na performance motora e diminuição gradual do movimento, sendo a fraqueza muscular um grande contribuinte para os distúrbios da marcha, afetando em última instância a capacidade laboral e a adaptabilidade ao ambiente.

As causas das quedas podem ser variadas e frequentemente estão associadas. Os fatores etiológicos podem ser classificados como intrínsecos, ou seja, decorrentes de alterações fisiológicas relacionadas ao envelhecimento, doenças e efeitos colaterais de medicamentos; e extrínsecos, que são circunstâncias sociais e ambientais de risco para o idoso. ${ }^{5}$ Os problemas com o ambiente serão mais perigosos quanto maior for o grau de vulnerabilidade do idoso e a instabilidade que este problema poderá causar. A maior parte das quedas de idosos não está vinculada a atividades perigosas (por exemplo, subir em escadas ou cadeiras), mas sim a atividades rotineiras. A maioria das quedas é da própria altura, no período diurno ${ }^{6}$ e ocorrem no próprio lar do idoso; a minoria, na rua ou na casa de parentes e amigos. ${ }^{5}$ Este estudo teve por objetivo revisar os fatores relacionados às quedas em idosos.

\section{A busca de fatores associados a quedas em idosos}

Esta revisão empregou, na estratégia de busca, as palavras-chave recomendadas por cada base de dados. A localização foi realizada nas seguintes bases de dados: The Cochrane Library, 
MEDLINE (via PubMed), LILACS e sites e bibliotecas on-line. O período de busca dos artigos foi de 1995 a 2011.

\section{Fatores de risco para quedas}

Quando avaliados do ponto de vista do indivíduo que cai, os fatores de risco associados a quedas podem ser classificados em intrínsecos (relativos ao indivíduo) ou extrínsecos (relativos ao meio ambiente em que o indivíduo circula).

\section{Fatores intrínsecos}

Dentre os fatores intrínsecos, as quedas em idosos se mantêm associadas com o sexo feminino, a idade avançada, o sedentarismo, o antecedente de quedas, a autopercepção de saúde ruim, as doenças crônicas e o maior número de medicamentos referidos para uso contínuo. A maioria das quedas ocorre entre idosos do sexo feminino (66\%), com idade média de 76 anos, no seu próprio lar (66\%). ${ }^{5}$

Além disso, observou-se que idosos separados ou divorciados apresentam elevada possibilidade de quedas. ${ }^{3} \mathrm{O}$ cuidado mútuo entre parceiros pode explicar a ocorrência reduzida de quedas entre aqueles que vivem com companheiro.

Uma maior chance de queda para o sexo feminino já foi indicada em vários estudos, mesmo controlando variáveis como idade, índice de massa corpórea e distúrbios de equilíbrio e marcha. As possíveis causas para explicar esse fenômeno permanecem ainda pouco esclarecidas. Sugeremse como causas a maior fragilidade das mulheres e a maior prevalência de doenças crônicas, além de uma maior exposição a atividades domésticas e comportamentos de risco. ${ }^{7,8}$ Provavelmente, as quedas ocorrem mais no sexo feminino também devido ao pior estado funcional, maior morbidade e maior presença de osteoartrose. ${ }^{9}$ As mulheres também apresentam maior perda de massa óssea por causa da redução do estrógeno a partir dos 40 anos de idade, contribuindo para deteriorar o seu estado funcional. ${ }^{10}$

A alteração da capacidade funcional parece ter um importante papel na fisiopatologia das quedas." Os idosos com baixa autoconfiança em realizar atividades do dia a dia, pelo medo de cair, tendem a ter um comprometimento progressivo em sua capacidade funcional ao longo do tempo.
Esse medo está também associado ao risco de quedas no futuro, mesmo em pessoas que nunca as sofreram. ${ }^{12}$

Desta forma, a institucionalização representa um fator de risco para quedas, já que a mudança do ambiente pode predispor a alterações psicológicas, cognitivas e funcionais relacionadas ao isolamento e à adaptação inadequada a um novo ambiente. ${ }^{13,14}$ A associação do declínio funcional e fragilidade tornam o idoso institucionalizado mais suscetível a novas quedas, comprometendo ainda mais sua independência.

Idosos de 75 a 84 anos que necessitam de ajuda para realização de atividades de vida diária (AVD) têm 14 vezes maior probabilidade de cair do que pessoas independentes. ${ }^{5} \mathrm{O}$ fato de precisar de ajuda ou deixar de realizá-las pode causar imobilidade e atrofia muscular, facilitando a queda. No estudo Epidoso, observou-se que idosas com quatro ou mais atividades físicas comprometidas apresentam uma probabilidade de $71,5 \%$ de já terem caído no ano anterior. ${ }^{8}$

Quando o idoso cai, há uma tendência à diminuição de suas atividades diárias, seja por medo de expor-se ao risco de queda, como por atitudes protetoras de familiares e cuidadores. A presença de queda prévia como fator de risco para quedas recorrentes foi descrita em vários estudos. Além disso, a possibilidade de ter experimentado uma queda anterior com consequência séria, como fratura, parece imputar ao idoso uma maior vulnerabilidade a novos episódios, independentemente da frequência deles. ${ }^{8}$ Mais de dois terços dos idosos que sofrem uma queda cairão novamente nos seis meses subsequentes.

A presença de doenças que ocasionem redução da capacidade física pode acarretar efeitos sobre o controle postural do indivíduo. As principais condições patológicas que predispõem à queda são: doenças cardiovasculares, neurológicas, osteomusculares, endocrinológicas, geniturinária, psiquiátricas e sensoriais.

A hipotensão postural, seja por etiologia cardiovascular, autonômica, medicamentosa, por desidratação ou imobilidade, é uma entidade de elevada prevalência e que pode ser causa direta de queda recorrente.

A cardiopatia pode estar presente em idosos institucionalizados, e a queda pode ser o primeiro sinal de um infarto agudo do miocárdio assintomá- 
tico. Certas arritmias como os bloqueios atrioventriculares, alterações do nó sinusal e bradicardias ou taquicardias graves também podem ocasionar quedas. ${ }^{15}$ Ainda existe controvérsia na literatura científica sobre associação entre hipertensão arterial sistêmica e quedas, uma vez que alguns estudos não encontraram esta associação, enquanto outros a relacionam à hipotensão postural causada pelo uso de anti-hipertensivos. ${ }^{4}$

Dentre as doenças neurológicas, a doença de Parkinson, por suas características de alteração de equilíbrio, postura e marcha, é uma etiologia potencial e frequente para quedas. As neuropatias periféricas podem contribuir para quedas por alteração da informação sensorial postural, independente do fator etiológico básico.

As síndromes demenciais podem levar à queda devido a alterações de marcha, como na hidrocefalia normobárica, ou pelo fato dos indivíduos não terem condições de alocar recursos cognitivos relacionados à atenção, julgamento e orientação visuoespacial, suficientes para o controle postural e adaptação ao ambiente. ${ }^{16}$ Por outro lado, alguns estudos não encontraram uma relação significativa entre queda e o deficit cognitivo. Acredita-se que, nestes casos, os idosos avaliados estão mais comprometidos cognitiva e funcionalmente, estando limitados a cadeiras de rodas ou suas camas.

A depressão também é uma condição de risco para quedas, em decorrência do efeito de medicações antidepressivas e sedativas, da alteração de atenção associada, do declínio da capacidade funcional, baixa autoconfiança, indiferença ao meio ambiente, reclusão e inatividade.

Distúrbios osteomusculares, como osteoartrose, que resultam em rigidez e artralgias, estão ligados à instabilidade no equilíbrio estático e na deambulação. A perda da força muscular consequente a estes distúrbios pode prejudicar a mobilidade, levando à dependência funcional e quedas. ${ }^{17}$

O deficit na acuidade visual, restrição do campo visual, aumento da suscetibilidade à luz, percepção de profundidade deficiente ou instabilidade na fixação do olhar são fatores relacionados à perda de equilíbrio. Além disso, a percepção subjetiva de diminuição da visão parece ser um fator de risco ainda mais importante. ${ }^{17,18}$ Isto pode estar relacionado ao fato de que o impacto da limitação visual sobre a capacidade funcional é mais expressivo do que simplesmente a presença de doenças visuais.

A relação de quedas e deficit auditivo não é tão clara, porém há evidências de que a diminuição da sensibilidade auditiva resulta em vertigens, dificulta o controle postural e a marcha, que pode interferir na capacidade de realizar tarefas cotidianas e maior risco de quedas.

$\mathrm{O}$ uso de medicamentos tem sido abordado em vários estudos como um dos principais fatores de risco para quedas. ${ }^{19} \mathrm{Em}$ um estudo, foi observado que $70 \%$ dos idosos faziam uso de algum tipo de medicação antes de cair, e o uso de muitos fármacos foi verificado em $42 \%$ deles. $^{5}$ Os medicamentos mais utilizados pelos idosos foram os anti-hipertensivos (34\%), seguidos por hipoglicemiantes (14\%). O uso de quatro ou mais drogas associadas aumenta o risco de queda, devido ao fato de haver forte interação entre elas ou ainda que o tratamento com vários fármacos pode traduzir uma condição de saúde mais precária. ${ }^{6}$

Medicações como benzodiazepínicos, neurolépticos, sedativos/hipnóticos, antidepressivos (especialmente os tricíclicos), diuréticos, antiarrítmicos, digoxina, anti-hipertensivos e antiparkinsonianos podem propiciar quedas. Isto muitas vezes ocorre porque essas drogas podem diminuir as funções motoras e causar sedação, diminuição do tempo de reação, fraqueza muscular, fadiga, vertigem ou hipotensão ortostática. ${ }^{20}$

$\mathrm{O}$ aumento no risco de quedas entre idosos em uso de benzodiazepínicos deve-se a duas propriedades desses medicamentos: atividade sedativa e bloqueio alfa-adrenérgico. A primeira seria responsável por alterações psicomotoras, enquanto a segunda aumentaria a probabilidade de hipotensão. Os agentes hipnótico-sedativos de meia-vida longa podem causar sedação residual durante o dia, tornando os idosos mais sujeitos a apresentar tonteiras, ataxia e confusão mental, levando ao maior risco de quedas e fraturas graves.

\section{Fatores extrínsecos}

As quedas são um resultado da interação dos fatores intrínsecos decorrentes das alterações fisiológicas e patológicas do processo de envelhecimento e dos fatores extrínsecos ligados aos riscos ambientais, tais como iluminação, superfície irregular ou escorregadia, presença de tapetes 
soltos, degraus altos ou estreitos ${ }^{21}$ e fatores comportamentais mediados pela percepção do espaço.

Muitas das causas das quedas estão relacionadas aos aspectos físicos ambientais, sendo os mais encontrados nos domicílios dos idosos o piso escorregadio, a presença de tapetes, a presença de objetos desordenados e os armários difíceis de alcançar. ${ }^{22}$ Outros fatores que também colaboram para a insegurança do idoso nos domicílios são: iluminação inadequada; interruptores inacessíveis; falta de corrimão nas escadas; degraus inadequados e sem sinalizações ou sem piso antiderrapante; falta de barras de apoio nos banheiros; assentos sanitários, camas e cadeiras de alturas inadequadas e sem apoiadores laterais; obstáculos no caminho, como móveis baixos e fios; presença de animais de estimação.

Alguns idosos, por não aceitarem suas limitações, se colocam em situações de risco, realizando atividades que resultam em quedas, como por exemplo: subir em uma escada dobrável para limpar um armário ou para pegar algum utensílio doméstico. Idosos que nunca caíram e com bom estado funcional também se colocam em situações de risco como entrar em uma sala quando está escura, não utilizar iluminação noturna e subir em banco para alcançar objetos. O uso inadequado de calçados também é responsável pelas quedas na população idosa.

Situações rotineiras como tomar banho, deambular, sentar ou levantar de cadeiras e camas podem ser responsáveis por diversos eventos de quedas entre os idosos.

A identificação dos fatores de risco causadores das quedas é uma importante estratégia para estabelecer programas de prevenção das mesmas.

Um ambiente pode ser considerado ideal para o idoso quando oferece segurança, facilita o desenvolvimento da sua funcionalidade, proporciona a estimulação cognitiva, facilita a mobilidade e a interação social, favorece a adaptação às mudanças e é aconchegante e familiar para o idoso. É importante ressaltar que as mudanças realizadas no domicílio devem ser realizadas com a autorização do idoso, pois sua casa e seus objetos possuem uma memória afetiva e qualquer alteração irá necessitar de uma reorganização interior para fornecer maior segurança.

\section{A marcha e o equilíbrio}

A marcha de idosos saudáveis pode ser caracterizada por meio de medidas temporais e espaciais. Uma das características que acompanham o envelhecimento é a redução da velocidade da marcha. A velocidade da marcha é determinada pelo comprimento da passada e pela cadência, sendo linear em indivíduos jovens e idosos saudáveis. $\mathrm{O}$ comprimento da passada para a população, em geral, é de aproximadamente 1,41 metros, sendo a média masculina 1,46 metros, e a feminina, de 1,28 metros. $^{23}$

A literatura sugere que a velocidade da marcha pode estar relacionada com a diminuição de força muscular e capacidade funcional da pessoa idosa. ${ }^{24}$ Idosos saudáveis podem aumentar sua velocidade de marcha pelo aumento do comprimento da passada e diminuição do ciclo de duração, ao passo que alguns idosos podem reduzir a velocidade da marcha na tentativa de obter uma marcha mais estável e segura.

Algumas doenças promovem alterações na capacidade do andar dos idosos. Estas alterações se apresentam de acordo com quatro categorias funcionais. São elas: deformidades, fraqueza muscular, falta de controle e dor. ${ }^{23}$ Cada categoria possui uma característica própria que pode levar a um prejuízo funcional. O reconhecimento dessas alterações permite uma avaliação criteriosa e diferenciada entre o que é deficit primário e o que é resultado de ações compensatórias.

\section{Controle postural e deficit de equilíbrio}

O processo natural do envelhecimento vem acompanhado de modificações estruturais e funcionais do organismo. As estruturas responsáveis pelo equilíbrio também sofrem modificações proporcionando grande impacto na vida do idoso, devido ao comprometimento da sua qualidade de vida. A instabilidade postural é uma das causas das quedas nos idosos e suas consequências são o aumento do nível de dependência e de fragilidade, institucionalização e morte nesta população. ${ }^{25}$

Para que ocorra o controle postural é necessário que os sistemas visual, vestibular e somatossensorial estejam íntegros, proporcionando a manutenção do centro de gravidade sobre a base de sustentação com respostas eficazes em situações estáticas e dinâmicas. ${ }^{26}$ 
No idoso, os sistemas responsáveis pela estabilidade postural estão afetados e diversas etapas do controle postural podem se encontrar suprimidas, diminuindo a capacidade de resposta e de compensação, levando a um aumento da instabilidade.

A visão é predominante sobre todos os sistemas sensoriais. Os indivíduos tendem a utilizar e confiar na visão para muitas funções simples e complexas do dia a dia que exigem controle coordenado. ${ }^{27}$

É por meio da visão que obtemos as informações sobre o ambiente que estamos. Também é através da visão que percebemos as diversas formas, tamanhos, cores, posições e movimentos de tudo o que está à nossa volta, permitindo o controle dos movimentos necessários para a execução de ações ou tarefas específicas em diversos ambientes.

É através do sistema visual que é possível manter o balanço natural dentro dos limites da base de apoio, informando como manter o alinhamento da cabeça e do tronco quando ocorre perturbação do centro de massa. As alterações visuais mais comuns que podem acometer o idoso são: catarata, glaucoma e degeneração macular senil.

O sistema vestibular é responsável pelas informações relativas à posição da cabeça em relação à gravidade, funciona como um sensor de gravidade, e aos movimentos lineares e rotatórios da cabeça. ${ }^{27}$

O sistema vestibular consiste em dois tipos de sensores do movimento, que sentem diferentes aspectos da posição e movimentos da cabeça, os canais semicirculares e órgãos otolíticos. Os canais semicirculares são responsáveis por detectar os movimentos de rotação da cabeça e são sensíveis a movimentos rápidos. Os órgãos otolíticos percebem as inclinações da cabeça quando os movimentos são lentos e suaves.

A alteração mais encontrada no idoso é a vertigem posicional paroxística benigna (VPPB), que é a síndrome mais diagnosticada em ambulatórios especializados em vertigem. A doença tem caráter benigno, mas sabe-se que a VPPB aumenta o risco de quedas em idosos. O paciente com VPPB relata crises de vertigem do tipo rotatória, com duração curta e forte intensidade, que podem vir acompanhadas de náuseas e vômitos. Essas cri- ses são desencadeadas por movimentos rápidos da cabeça e os movimentos mais frequentes são: levantar da cama pela manhã, deitar e virar na cama, estender o pescoço para olhar para o alto e flexionar o pescoço para olhar para baixo. A partir do diagnóstico, o tratamento é eficaz, realizado através da reabilitação vestibular e medicamentos.

O sistema somatossensorial é responsável pelas informações proprioceptivas, especialmente aquelas associadas às articulações e músculos axiais, que fornecem informações sobre os movimentos e posições do corpo. Também são importantes as informações oriundas das regiões plantares. Os receptores somatossensitivos incluem os fusos musculares e os órgãos tendinosos de Golgi (sensíveis ao comprimento e à tensão dos músculos, respectivamente), receptores articulares e mecanorreceptores cutâneos, que fornecem informações sobre a orientação do corpo, com base na superfície de apoio. Estes receptores - ou receptores de estímulos de pressão - informam como e onde estamos pisando. ${ }^{27}$

Os idosos necessitam das informações sobre o ambiente para manter o controle postural, porém, o processo de envelhecimento é responsável por apresentar uma diminuição da sensibilidade desses receptores na detecção das mudanças de posição e também na diminuição da sensibilidade cutânea ao toque, à vibração e à capacidade de discriminação entre dois pontos.

\section{O conflito visual e o equilíbrio}

As condições ambientais determinam a qualidade das informações sensoriais e quais informações serão usadas para a manutenção do equilíbrio. O conflito sensorial pode ocorrer quando as informações dos sistemas sensoriais se encontram desajustadas. Ambientes conflituosos indicam a presença e a ausência de movimento ao mesmo tempo.

O sistema nervoso central deve ser capaz de selecionar as informações sensoriais sobre a verdadeira orientação do corpo para promover uma resposta motora adequada. Para isso, o sistema de controle postural atribui maior peso a um tipo de informação sensorial do que a outras, dependendo do ambiente onde a tarefa está sendo realizada.

O indivíduo dá preferência para cada um dos sistemas sensoriais de acordo com a importância da informação para o controle postural. Um bom 
exemplo é quando um indivíduo não perde o equilíbrio quando está em um ambiente escuro, onde as informações visuais estão restritas, provavelmente porque dará preferência às informações vestibulares e somatossensitivas. ${ }^{28}$

\section{Conclusão}

As quedas em idosos são eventos multifatoriais, e o conhecimento de seus fatores de risco é importante para nortear o planejamento de medidas preventivas. Os objetivos de tais medidas, tanto do ponto de vista de intervenções individuais quanto do de políticas públicas são evitar as quedas ou reduzir seu número, além de preservar a funcionalidade e melhorar a qualidade de vida.

\section{Referências}

1. Kay PD, Tideiksaar R. Quedas e distúrbios de marcha. In: Abrams WB, Berkow R. Manual Merck de Geriatria. São Paulo: Ed. Roca; 1995.

2. Sociedade Brasileira de Geriatria e Gerontologia, Buksman S, Vilela ALS, Pereira SRM, Lino VS, Santos VH. Projeto Diretrizes. Quedas em idosos: prevenção. São Paulo: Associação Médica Brasileira, Conselho Federal de Medicina; 2001.

3. Siqueira AB, Cordeiro RC, Perracini MR, Ramos LR. Impacto funcional da internação hospitalar de pacientes idosos. Rev Saúde Pública. 2004;38(5):68794.

4. Masud T, Morris RO. Epidemiology of falls. Age Ageing. 2001;30(S4):3-7.

5. Fabrício SCC, Rodrigues RAP, Costa MJ. Causas e consequências de quedas de idosos atendidos em hospital público. Rev Saúde Pública. 2004;38(1):93-9.

6. Coutinho ESF, Silva SD. Uso de medicamentos como fator de risco para fratura grave decorrente de queda em idosos. Cad Saúde Pública. 2002;18(5):1359-66.

7. Fried LP, Tangen CM, Walston J, Newman AB, Hirsch $\mathrm{C}$, Gottdiener J, et al. Frailty in older adults: evidence for a phenotype. J Gerontol A Biol Sci Med Sci. 2001;56(3):M146-56.

8. Perracini MR, Ramos LR. Fatores associados a quedas em uma coorte de idosos residentes na comunidade. Rev Saúde Pública. 2002;36(6):709-16.

9. Gac H, Marin PP, Castro S, Hoyl T, Valenzuela E. Caídas en adultos mayores institucionalizados: descripción y evaluación geriátrica. Rev Méd Chile. 2003;131(8):887-94.

10. Lebrão ML, Laurenti R. Saúde, bem-estar e envelheci- mento: o estudo SABE no município de São Paulo. Rev Bras Epidemiol. 2005;8(2):127-41.

11. Paula FL, Fonseca MJM, Oliveira RVC, Rozenfeld S. Perfil de idosos com internação por quedas nos hospitais públicos de Niterói (RJ). Rev Bras Epidemiol. 2010;13(4):587-95.

12. Lopes KT, Costa DF, Santos LF, Castro DP, Bastone AC. Prevalência do medo de cair em uma população de idosos da comunidade e sua correlação com mobilidade, equilíbrio dinâmico, risco e histórico de quedas. Rev Bras Fisioter. 2009;13(3):223-9.

13. Perracini MR. Prevenção e manejo de quedas. In: Ramos LR, coordenação. Guia de geriatria e gerontologia. Barueri: Manole; 2005. p. 193-208.

14. Santos MLC, Andrade MC. Incidência de quedas relacionada aos fatores de riscos em idosos institucionalizados. Rev Baiana Saúde Pública. 2005;29(1):57-68.

15. Brito FC, Costa SMN. Quedas. In: Papaleo Netto M, Brito FC. Urgências em geriatria. São Paulo: Atheneu; 2001. p. 323-35.

16. Carvalho AM, Coutinho ESF. Demência como fator de risco para fraturas graves em idosos. Rev Saúde Pública. 2002;36(4):448-54.

17. Menezes RL, Bachion MM. Estudo da presença de fatores de riscos intrínsecos para quedas em idosos institucionalizados. Ciênc Saúde Coletiva. 2008;13(4):1209-18.

18. Alvares LM, Lima RC, Silva RA. Ocorrência de quedas em idosos residentes em instituições de longa permanência em Pelotas, Rio Grande do Sul, Brasil. Cad. Saúde Pública. 2010;26(1):31-40.

19. Secoli SR. Polifarmácia: interações e reações adversas no uso de medicamentos por idosos. Rev Bras Enferm. 2010;63(1):136-40.

20. Coutinho ESF, Silva SD. Uso de medicamentos como fator de risco para fratura grave decorrente de queda em idosos. Cad Saúde Pública 2002; 18:1359-66.

20. Chaimowicz F, Ferreira TJXM, Miguel DFA. Use of psychoactive drugs and related falls among older people living in a community in Brazil. Rev Saúde Pública. 2000;34(6):631-5.

21. Kallin K, Lundin-Olsson L, Jensen J, Nyberg L, Gustafson Y. Predisposing and precipitating factors for falls among older people in residential care. PublicHealth. 2002;116(5):263-71.

22. Messias MG, Neves RF. A influência de fatores comportamentais e ambientais nas quedas em idosos. Rev Bras Geriatr Gerontol. 2009;12(2):275-82.

23. Perry J. Análise de Marcha - Volume 2 - Marcha Patológica. São Paulo: Ed. Manole; 2004. 210 p.

24. Callisaya ML, Blizzard L, Schmidt MD, McGinley 
JL, Srikanth VK. Ageing and gait variability - a population-based study of older people. Age Ageing. 2010;39(2):191-7.

25. Alexander NB. Postural control in older adults. J Am Geriatr Soc. 1994;42(1):93-108.

26. Ruwer SL, Rossi AG, Simon LF. Equilíbrio no idoso.

Recebido: 08/03/2013.

Revisado: 26/10/2013.

Aprovado: 29/11/2013.
Rev Bras Otorrinolaringol. 2005;71(3):298-303.

27. Tedeiksaar R. As Quedas na Velhice. Prevenção e Cuidados. $2^{\text {a }}$ ed. São Paulo: Andrei; 2003. 438 p.

28. Ricci NA, Gazolla JM, Coimbra IB. Sistemas sensoriais no equilíbrio corporal de idosos. Arq Bras Ciênc Saúde. 2009;34(2):94-100.

\section{Daniele A. Lima}

Serviço de Geriatria. Hospital Federal dos Servidores do Estado do Rio de Janeiro. Rio de Janeiro, RJ, Brasil.

\section{Vania O. B. Cezario}

Serviço de Geriatria Prof. Mario A. Sayeg. Policlínica Piquet Carneiro. Universidade do Estado do Rio de Janeiro. Rio de Janeiro, RJ, Brasil. 